\section{Fatigue, alcohol and performance impairment}

Reduced opportunity for sleep and Rreduced sleep quality are frequently related to accidents involving shift-workers ${ }^{1-3}$. Poor-quality sleep and inadequate recovery leads to increased fatigue, decreased alertness and impaired performance in a variety of cognitive psychomotor tests ${ }^{4}$. However, the risks associated with fatigue are not well quantified. Here we equate the performance impairment caused by fatigue with that due to alcohol intoxication, and show that moderate levels of fatigue produce higher levels of impairment than the proscribed level of alcohol intoxication.

Forty subjects participated in two counterbalanced experiments. In one they were kept awake for 28 hours (from 8:00 until 12:00 the following day), and in the other they were asked to consume 10-15 g alcohol at 30-min intervals from 8:00 until their mean blood alcohol concentration reached $0.10 \%$. We measured cognitive psychomotor performance at half-hourly intervals using a computer-administered test of hand-eye coordination (an unpredictable tracking task). Results are expressed as a percentage
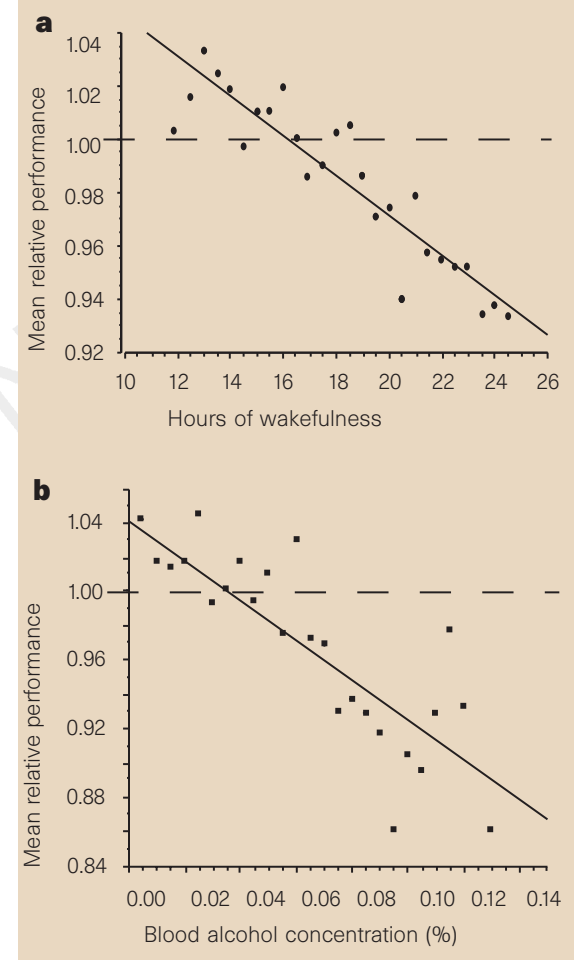

Figure 1 Scatter plot and linear regression of mean relative performance levels against: a, time, between the tenth and twenty-sixth hour of sustained wakefulness $\left(F_{1,24}=132.9, \quad P<0.05, R^{2}=0.92\right)$; and $\mathbf{b}$, blood alcohol concentrations up to $0.13 \%$, $\left(F_{1,24}=54.4, P<0.05, R^{2}=0.69\right)$. of performance at the start of the session.

Performance decreased significantly in both conditions. Between the tenth and twenty-sixth hours of wakefulness, mean relative performance on the tracking task decreased by $0.74 \%$ per hour. Regression analysis in the sustained wakefulness condition revealed a linear correlation between mean relative performance and hours of wakefulness that accounted for roughly $90 \%$ of the variance (Fig. 1a).

Regression analysis in the alcohol condition indicated a significant linear correlation between subject's mean blood alcohol concentration and mean relative performance that accounted for roughly $70 \%$ of the variance (Fig. 1b). For each $0.01 \%$ increase in blood alcohol, performance decreased by $1.16 \%$. Thus, at a mean blood alcohol concentration of $0.10 \%$, mean relative performance on the tracking task decreased, on average, by $11.6 \%$.

Equating the two rates at which performance declined (percentage decline per hour of wakefulness and percentage decline with change in blood alcohol concentration), we calculated that the performance decrement for each hour of wakefulness between 10 and 26 hours was equivalent to the performance decrement observed with a $0.004 \%$ rise in blood alcohol concentration. Therefore, after 17 hours of sustained wakefulness (3:00) cognitive psychomotor performance decreased to a level equivalent to the performance impairment observed at a blood alcohol concentration of $0.05 \%$. This is the proscribed level of alcohol intoxication in many western industrialized countries. After 24 hours of sustained wakefulness (8:00) cognitive psychomotor performance decreased to a level equivalent to the performance deficit observed at a blood alcohol concentration of roughly $0.10 \%$.

Plotting mean relative performance and blood alcohol concentration 'equivalent' against hours of wakefulness (Fig. 2), it is clear that the effects of moderate sleep loss on performance are similar to moderate alcohol intoxication. As about $50 \%$ of shiftworkers do not sleep on the day before the first night-shift ${ }^{5}$, and levels of fatigue on subsequent night-shifts can be even higher ${ }^{6}$, our data indicate that the performance impairment associated with shift-work could be even greater than reported here.

Our results underscore the fact that relatively moderate levels of fatigue impair performance to an extent equivalent to or greater than is currently acceptable for

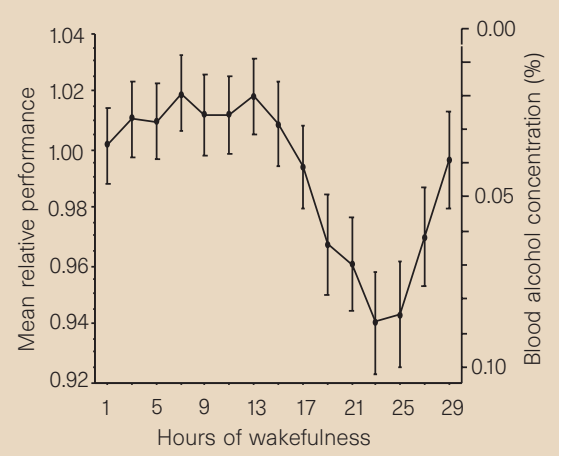

Figure 2 Performance in the sustained wakefulness condition expressed as mean relative performance and the percentage blood alcohol concentration equivalent. Error bars \pm s.e.m.

alcohol intoxication. By expressing fatiguerelated impairment as a 'blood-alcohol equivalent', we can provide policy-makers and the community with an easily grasped index of the relative impairment associated with fatigue.

\section{Drew Dawson}

The Centre for Sleep Research,

University of South Australia,

The Queen Elizabeth Hospital,

Woodville, 5011 South Australia

e-mail:ddawson@tqehsmtp.tqeh.sa.gov.au

\section{Kathryn Reid}

Department of Obstetrics and Gynaecology, University of Adelaide,

The Queen Elizabeth Hospital, Woodville, 5011 South Australia

\section{Mitler, M. et al. Sleep 11, 100-109 (1988). \\ 2. Leger, D. Sleep 17, 84-93 (1994). \\ 3. Akerstedt, T., Czeisler, C., Dinges, D. F. \& Horne, J. A. J. Sleep Res. 3, 195 (1994). \\ 4. Harrington, J. Shiftwork and Health: A Critical Review of the Literature. Report to the Medical Advisory Service, UK Health and Safety Executive (H. M. Stationery Off., London, 1978). \\ 5. Knauth, P. \& Rutenfranz, J. in Advances in the Biosciences Vol. 30. Night and Shiftwork. Biological and Social Aspects. (eds Reinberg, A., Vieux, N. \& Andlauer, P) 161-168 (Pergamon, Oxford, 1980). \\ 6. Tilley, A., Wilkinson, R. \& Drud, M. in Advances in the Biosciences Vol. 30. Night and Shiftwork. Biological and Social Aspects. (eds Reinberg, A., Vieux, N. \& Andlauer, P.) 187-196 (Pergamon, Oxford, 1980). \\ Entropy difference between crystal phases}

In a recent Letter ${ }^{1}$, Woodcock reported the results of a molecular dynamics study in which he claims to have finally determined the free-energy difference between the hexagonal close-packed (h.c.p.) and facecentred cubic (f.c.c.) phases of a crystal of (classical) hard spheres. Woodcock reports a small positive difference in the reduced Gibbs free-energy, which is equivalent to a difference in the reduced Helmholtz freeenergy of $\Delta F^{*} \equiv\left(F_{\mathrm{hcp}}-F_{\mathrm{fcc}}\right) / R T=0.005(1)$ at the melting density ( $R$ is the gas constant, $T$ is the absolute temperature, and the num- 
ber in parentheses is the estimated error in the last digit). As Woodcock correctly points out, the calculation of the relative stability of the f.c.c. and h.c.p. phases of hard spheres is a long-standing problem in statistical physics. Attempts to resolve it date back to the work of Alder, Hoover and colleagues $^{2-5}$, and most recently, a direct simulation by Frenkel and Ladd $^{6}$, obtaining the bounds of Helmholtz free-energy of $-0.001 \leqslant \Delta F^{*} \leqslant 0.002$. Woodcock's estimate is incompatible with this latter result.

To resolve this issue, we made accurate calculations of the free-energy difference between h.c.p. and f.c.c. hard-sphere crystals both at the melting density $(73.6 \%$ of the density of regular close packing) and at close packing, using two different methods. We find that $\Delta F^{*}=0.0009(2)$ at melting, a result that is quite consistent with the earlier work, but is five times smaller than Woodcock's estimate. Woodcock does not explain how he arrives at an error estimate of $20 \%$ - our work suggests that the numerical error in his result must have been four times larger than the entire h.c.p. - f.c.c. free-energy difference.

Nevertheless, we do agree with the sign of Woodcock's estimate - the f.c.c. crystal is indeed more stable than the h.c.p. crystal. This might explain the tendency towards f.c.c. packing seen in some experimental studies of hard-sphere colloids ${ }^{7}$. In one set of simulations, we used the 'Einstein-crystal' method ${ }^{6,8}$, simulating crystals of 12,096 hard spheres (slightly larger than the largest system studied by Woodcock), and computed the Helmholtz free-energies of the two phases using a 20-point Gauss-Legendre quadrature. Every point in this quadrature involved a Monte Carlo simulation of $10^{5}$ trial moves per particle, excluding equilibration. We find that the free-energy difference between h.c.p. and f.c.c. at melting is $\Delta F^{*}=0.00087(20)$, and at close packing $\Delta F^{*}=0.00094(30)$. The statistical error was computed on the basis of the variance in the block averages of the individual Monte Carlo runs ${ }^{9}$.

We also performed simulations using a new 'multi-hamiltonian' method (S.-C. M. and D. A. H., manuscript in preparation) that directly equilibrates the h.c.p. and f.c.c. hard-sphere crystals with each other by a set of intermediate states with different interactions but essentially the same free-energy. These latter simulations were done on much smaller samples (64 to 512 spheres) and obtained essentially the same freeenergy differences (for 512 spheres, $\Delta F^{*}=0.00085(10)$ near melting, and $0.0011(2)$ at close packing) as the 'Einsteincrystal' simulations, with comparable statistical errors. Statistically significant finite-size effects were detected only for the smallest size (64 spheres) near melting, where $\Delta F^{*}$ dropped to near zero.
In any event, our result for the f.c.c.-h.c.p. free-energy difference for large hard-sphere crystals at melting is much closer to $\Delta F^{*}=0$, proposed almost 30 years ago by Alder and co-workers, than to the recent estimate by Woodcock.

\section{P. G. Bolhuis, D. Frenkel}

FOM Institute for Atomic and Molecular Physics,

Kruislaan 407, 1098 SJ Amsterdam,

The Netherlands

Siun-Chuon Mau, David A. Huse

Department of Physics,

Princeton University,

Princeton, New Jersey 08544, USA

Woodcock replies - I reported the discovery a substantial area of pressure difference $(\Delta P)$ between the f.c.c. and h.c.p. singleoccupancy-cell models, which arises from a difference in order-disorder transition pressures. The result was a free-energy difference in favour of f.c.c., corresponding to an entropy difference $0.005 N k_{\mathrm{B}}$, over the range $V=1.00 \mathrm{N \sigma}^{3}$ to $1.25 \mathrm{N \sigma}^{3}$, with a generous uncertainty $( \pm 0.001)$, estimated by integrating the standard deviations of subaverages of $\Delta P$ for individual data points. Extension of the computations on either side of the phase transition have since revealed a tail in the pressure difference for $V>1.25 N \sigma^{3}$ in favour of h.c.p. There is also a weak pressure difference for volumes below melting. I have now obtained more accurate data for these tails, including new data points on both sides of the singleoccupancy-cell phase transition (Fig. 1).

I did not originally calculate the pressure difference in the stable crystal range, relying on earlier findings that $\Delta P$ up to melting was not detectable by molecular dynamics computation $^{2}$, and that these showed the two crystals to have indistinguishable crystal constants $\mathrm{C}_{0}$ and $\mathrm{C}_{1}$ (ref. 4). Consequently I assumed no difference between the Gibbs and Helmholtz free-energies in the stable

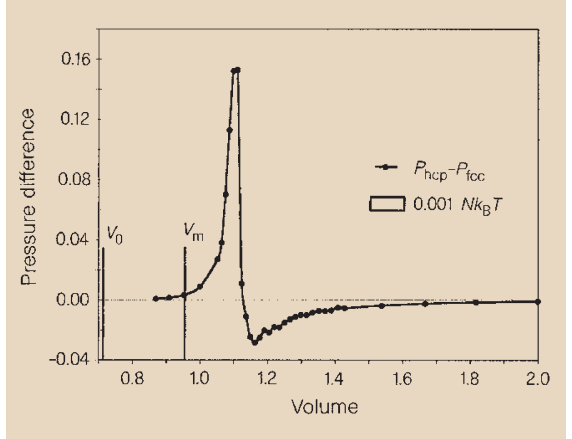

Figure 1 Latest molecular dynamic data for the pressure difference as a function of volume at constant temperature, $\Delta P\left(V_{T}\right.$, between the h.c.p. and f.c.c. single-occupancy-cell crystal structures for hard spheres; $V_{0}$ is the close-packed crystal volume and $V_{\mathrm{m}}$ is the volume at melting. The area under this curve is the Helmholtz free-energy difference between the two crystal structures at close packing in units of $N k_{\mathrm{B}} T$. crystal range.

A detectable pressure difference between f.c.c. and h.c.p. crystals below melting, however, has now been computed, both by R. Speedy (personal communication) and myself. This small pressure difference means that the entropy difference at constant volume - which equals the Helmholtz free-energy difference for hard spheres - is not the same as the Gibbs freeenergy difference, which determines the stable crystal structure at freezing. However, the correction is small, $\sim 0.000015 N k_{\mathrm{B}} T$.

At the melting volume $\left(V_{\mathrm{m}}\right)$ of $0.96 N \sigma^{3}$, I calculate the pressure difference to be $0.0030(5) k_{\mathrm{B}} T / \sigma^{3}(N=12,000)$. Alder et al. ${ }^{3}$ adopted too large a value for $\Delta P_{\mathrm{m}}$ $\left(0.02 k_{\mathrm{B}} T / \sigma^{3}\right)$, and further guessed wrongly that the absolute difference decreased linearly with density to zero at $V_{0}$. In fact they estimated the Helmholtz free-energy difference $\left(\Delta F_{\mathrm{m}}-\Delta F_{0}\right)$ to be $0.002 N k_{\mathrm{B}} T$ in favour of f.c.c. My data (Fig. 1) show that the pressure difference found at melting actually decreases to negligible values more rapidly, and that the change in free-energy difference between close packing and melting is of the order $0.0003 N k_{\mathrm{B}} T$. The closeness of the result of Alder et al. to any of the present results, or indeed to zero, is therefore an irrelevance.

The Einstein-crystal method ${ }^{10}$ (used both by Frenkel and Ladd ${ }^{6}$ and here by Bolhuis and Frenkel), the multi-hamiltonian method and the Hoover-Ree single-occupancy-cell method, if accurately implemented, should all give the correct answer. I am still working on this problem, but the latest result for the Helmholtz free-energy difference between the h.c.p. and f.c.c. structures (f.c.c. having the lower freeenergy) at close packing gives:

$\Delta F_{0}=\int_{V_{0}}^{\infty}\left(P_{\mathrm{hcp}}-P_{\mathrm{fcc}}\right) \mathrm{d} V=0.0026 \pm 0.001 N k_{\mathrm{B}} T$.

The change in Helmholtz free-energy difference between close-packing and the melting volume amounts to only $0.0003(1) N k_{\mathrm{B}} T$, as shown by the tiny, positive area in $\Delta P(V)_{T}$ up to the melting volume $\left(V_{\mathrm{m}}\right)$ (see Fig. 1). Hence, the Helmholtz free-energy difference at the melting volume is $\Delta F_{\mathrm{m}}=0.0023(10) N k_{\mathrm{B}} T$. There remains a quantitative disagreement between my result and the other two methods, but my original conclusion that the f.c.c. phase is everywhere the more stable crystal phase for hard spheres is confirmed by all the new results. It is also gratifying that the result for the tiny free-energy difference between close packing and melting show a remarkable consistency, within the error bars, by all three methods.

\section{V. Woodcock}

Department of Chemical Engineering,

University of Bradford, Bradford,

West Yorkshire BD7 1DP, UK 
1. Woodcock, L. V. Nature 385, 141-143 (1997).

2. Alder, B. J., Hoover, W. G. \& Young, D. A. J. Chem. Phys. 49, 3688-3696 (1968)

3. Alder, B. J., Carter, B. P. \& Young, D. A. Phys. Rev. 183, 831-833 (1969).

4. Alder, B. J., Young, D. A., Mansigh, M. R. \& Salsburg, Z. W. J. Comp. Phys. 7, 361-366 (1971).

5. Young, D. A. \& Alder, B. J. J. Chem. Phys. 60, 1254-1267 (1974)

6. Frenkel, D. \& Ladd, A. J. C. J. Chem. Phys. 81, 3188-3193 (1984).

7. Pusey, P. N. et al. Phys. Rev. Lett. 63, 2753-2756 (1989).

8. Bolhuis, P. G. \& Frenkel, D. J. Chem. Phys. 106, 666-687 (1997)

9. Frenkel, D. \& Smit, B. Understanding Molecular Simulation (Academic, Boston, 1996).

10. Broughton, J. Q. \& Gilmer, G. H. J. Chem. Phys. 79, 5095-5104 (1983).

\section{Metallothionein in snail}

\section{$\mathrm{Cd}$ and Cu metabolism}

Terrestrial snails tolerate elevated concentrations of cadmium and copper, accumulating both metals in their soft tissues ${ }^{1}$. The snails are able to inactivate the toxic cadmium while meeting their metabolic requirement for copper. Here we report evidence for the metabolic discrimination between the two metals based on the existence of distinct metallothionein isoforms, one dedicated to cadmium detoxification and another to copper regulation.

Even snails living in relatively unpolluted environments have the exceptional abili-

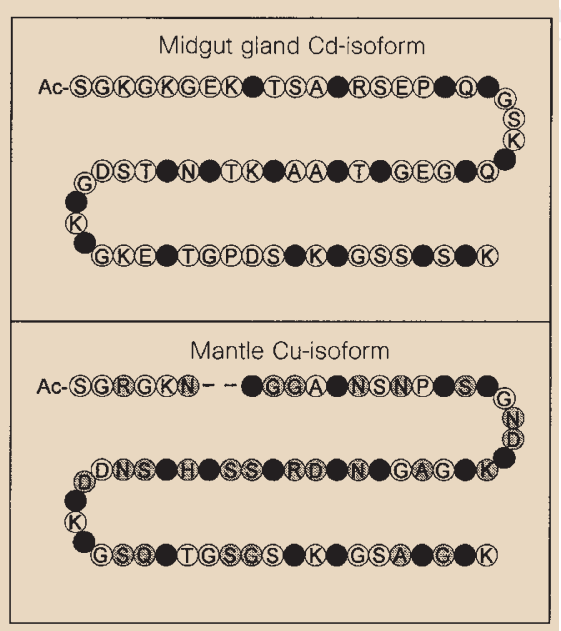

Figure 1 Primary structures of the cadmium- and copper-binding metallothionein isoforms from the midgut gland and mantle of $\mathrm{H}$. pomatia. Residues are indicated using single-letter code, with cysteines in black. The $\mathrm{N}$ termini are acetylated (Ac). Substituted residues are indicated in grey in the copper-binding isoform. The cadmium-binding isoform was purified and sequenced as described earlier $^{5}$. The copper-binding isoform was purified from mantle tissue by combined gel permeation, ionexchange chromatography, and reversed-phase HPLC. After endoproteinase digestion (trypsin, Lys-C and $\operatorname{Arg}-\mathrm{C}$ ) of $S$-methylated protein, peptides were sequenced by collision-induced tandem mass spectrometry (API III, Sciex, Canada) using argon as the collision gas $\left(4 \times 10^{14}\right.$ molecules $\left.\mathrm{cm}^{-2}\right)$. ty to concentrate cadmium - more than many other terrestrial invertebrates - in the midgut gland ${ }^{2}$. In contrast, copper, which is an essential constituent of the oxygen-carrying protein haemocyanin ${ }^{3,4}$, is predominantly present in the snail's foot and mantle ${ }^{1}$. The concentration of copper is kept constant, with animals quickly eliminating any excess that may have entered the tissue after environmental exposure ${ }^{1}$. We have recently isolated and characterized two metallothionein isoforms from terrestrial helicid species, differentially involved in the handling of cadmium and copper.

One of these isoforms is present in the midgut gland of terrestrial snails. We identified it as a class-I metallothionein ${ }^{5}$ with a typically low molecular mass $\left(6.62 \times 10^{6}\right.$; $6,620 \mathrm{~K})$, containing 66 amino acids, 18 of which are cysteines. Its amino-terminal serine is acetylated (Fig. 1). This isoform occurs in several variants in helicid snails, including Helix pomatia and Arianta arbustorum ${ }^{6,7}$.

The function of this isoform is the detoxification of cadmium, binding $85-95 \%$ of all cadmium accumulated in the snail soft tissues. The cadmium-binding metallothionein isoform can be isolated in a pure form from the midgut gland of metal-exposed snails, and has a molar metal ratio of $\mathrm{Cd}: \mathrm{Cu}: \mathrm{Zn}$ of $100: 2: 6.6$ in the native protein and a stoichiometry of six cadmium atoms per protein molecule (determined by spectrophotometric metal titration under nitrogen atmosphere). Its concentration increases linearly with increasing cadmium concentrations in the midgut gland (Fig. 2a).

We have recently isolated another isoform from the mantle of Helix pomatia. Apart from its acetylated amino-terminal serine, the primary structure is very different to the cadmium-binding metallothionein. It has a different molecular mass $(6,247 \mathrm{~K})$, and many amino-acids between the conserved cysteine residues have been substituted (Fig. 1). In vivo, this isoform is almost exclusively conjugated with copper, with a molar metal ratio of $\mathrm{Cu}: \mathrm{Cd}: \mathrm{Zn}$ of 100:1:6. We determined the stoichiometry using combined atomic absorption spectrophotometry, amino-acid analysis and electrospray mass spectrometry, as roughly six copper atoms per protein molecule.

The concentration of the mantle isoform and its exclusive preference for copper remain unaffected when snails are exposed to cadmium (Fig. 2b), even if this metal is injected into the mantle tissue. In this case, most of the administered cadmium is quickly eliminated from the mantle and redistributed to the midgut gland, but virtually none of the metal becomes bound to the copper-specific metallothionein isoform. In addition, the concentration of this isoform is barely affected by exposure of animals to large amounts of copper (Fig. $2 b)$. Our results indicate that the metalloth-

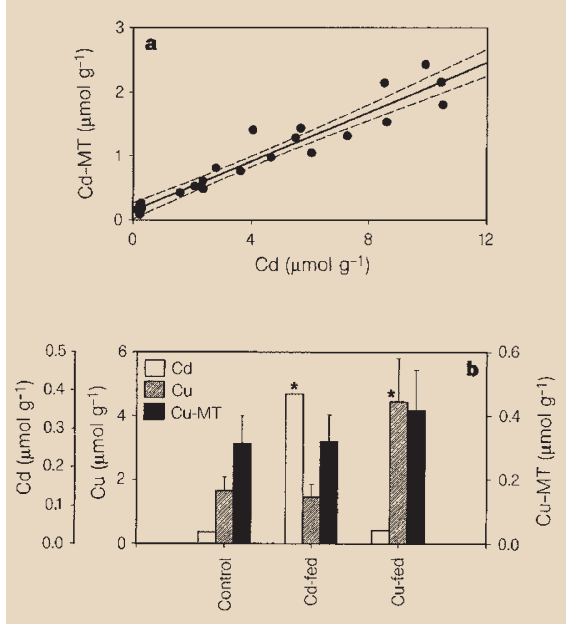

Figure 2 a, Linear relationship (bold line; regression coefficient $r=0.96)$, with $95 \%$ confidence limits (hatched lines) between molar concentrations (on a tissue dry-mass basis) of $\mathrm{Cd}$ and $\mathrm{Cd}$-metallothionein (Cd-MT) in the midgut gland of $\mathrm{H}$. pomatia fed on a Cd-enriched diet (3.5-955 $\mu \mathrm{g} \mathrm{Cd}$ per g dry mass) for 14 days. b, Molar concentrations of $\mathrm{Cd}$, $\mathrm{Cu}$, and $\mathrm{Cu}$-metallothionein (Cu-MT) in the mantle of $\mathrm{H}$. pomatia after feeding the animals on uncontaminated salad (control) or on Cd-enriched (Cd-fed; $260 \mu \mathrm{g}$ per g dry weight) or Cu-enriched diets (Cufed; $530 \mu \mathrm{g}$ per $\mathrm{g}$ dry weight) for 14 days. Mean concentration \pm s.d. $(n=7)$. Asterisks indicate significant differences $(P<0.01)$ from control values (Student's $t$-test). Concentrations of $\mathrm{Cd}$-metallothionein and $\mathrm{Cu}$-metallothionein were determined by modified $\mathrm{Cd}$ - and Cu-saturation assays ${ }^{11}$ (removing $\mathrm{Cu}$ from the holo-metallothionein with ammoniumtetrathiomolybdate). Similar results (not shown) were obtained after injecting $\mathrm{Cd}$ and $\mathrm{Cu}$ into mantle tissue.

ionein isoform in the mantle of terrestrial snails is concerned with the regulation of copper, probably in connection with haemocyanin synthesis (as the gastropod mantle is an important site of production of this copper-containing protein $)^{8}$.

Until now, the simultaneous handling of different metals by metallothioneins has been explained on the basis of metal-specific preferences of the two metal-binding domains of the molecule ${ }^{9,10}$. The existence of specific metallothionein isoforms dedicated to cadmium detoxification and copper regulation in snails suggests an alternative model to explain the mechanisms of multifunctionality in these proteins.

\section{Reinhard Dallinger}

\section{Burkhard Berger}

Institut für Zoologie und Limnologie,

(Abteilung Ökophysiologie),

Universität Innsbruck, Technikerstrasse 25,

A-6020 Innsbruck, Austria

\section{Peter Hunziker}

Jeremias H. R. Kägi

Biochemisches Institut der Universität Zürich,

Winterthurerstrasse 190,

CH-8057 Zürich, Switzerland 